\title{
Definition and scoring system of molar incisor hypomineralization: A review
}

\author{
Eman Allam $^{1,2}$, Ahmed Ghoneima ${ }^{1}$ and Katherine Kula ${ }^{\text {* }}$ \\ ${ }^{1}$ Department of Orthodontics and Oral Facial Genetics, Indiana University School of Dentistry, Indianapolis, IN, USA \\ ${ }^{2}$ Oral and Dental Research Division, National Research Centre, Cairo, Egypt
}

\begin{abstract} clinicians and researchers. is required.

\section{Introduction}

Molar incisor hypomineralization (MIH) is the term used to describe a special pattern of enamel defects. The terminology was first used by Weerheijm et al. [1] describing developmental defects affecting first permanent molars and permanent incisors, ranging from distinct, isolated, white, yellowish, or brown-colored demarcated opacities to severe post eruptive structural breakdown. Shortly after MIH terminology was proposed, the European Academy of Paediatric Dentistry (EAPD) announced criteria specifically aiming at diagnosing and recording $\mathrm{MIH}[2,3]$.
\end{abstract}

Objectives: A definition and classification system for molar incisor hypomineralization (MIH) was first suggested by the European Academy of Pediatric Dentistry (EAPD) in 2003. Since then, there has been growing interest in these mineralization defects due to esthetic and cariogenic susceptibility concerns. The aim of this review was to evaluate studies on $\mathrm{MIH}$ in an attempt to provide evidence for consistency and standardization of $\mathrm{MIH}$ definition and severity scoring reporting by

Methods: Included were studies (research papers and reviews) focusing on MIH affected populations and containing a definition and scoring system. Only English language articles published in the period 2001 to 2015 were considered. Study selection and data extraction were performed by two independent investigators.

Results: More than half of the publications used the EAPD definition and fewer than half of the prevalence reports have either used the EAPD criteria or the developmental defects of enamel (DDE) index as their classification system. Others have either incorporated modifications or created their own scoring criteria. The lack of standardized definition and heterogeneity of scoring systems used might explain the considerable variability in prevalence figures.

Conclusion: In order to better define the diagnostic criteria and the treatment needs for those patients, a globally accepted standardized protocol for scoring system

Information available on the exact etiology of MIH is limited. A combination of factors that may affect the ameloblasts resulting in abnormal enamel formation has been suggested in most studies. Systemic or environmental insults during the maturation stages of enamel development as well as the possibility of genetic predisposition of ameloblasts to environmental stressors have been reported [46]. Some authors suggest that MIH may be an autosomal recessive condition or an unrecognized form of localized amelogenesis imperfecta. Others have indicated that there is a possible association between $\mathrm{MIH}$ and variants in genes related to enamel formation such as AMBN, TUFT1, and TFIP11 [7,8]. A genome-wide association study identified the SCUBE1 gene on chromosome 22 as a potential genetic locus for MIH [9].

There is wide disparity in the reported prevalence figures of MIH. The majority of reports are from European populations, with prevalence rates ranging from $5.9 \%$ to $38 \%[10,11]$. Other Non-European prevalence data also demonstrate extreme variations with a prevalence rate as low as $2.8 \%$ in Hong Kong [12] and a rate as high as $40.2 \%$ in
Brazilian children [13]. The extensive variation in the described rates is suggested to be either due to actual differences in $\mathrm{MIH}$ prevalence between different populations, variations in age cohorts, masking of MIH by other conditions, or most importantly, due to the different clinical examination protocols used to define this condition and the non-uniform methods of classification and scoring indices [14-17]. The objective of the current review was to systematically evaluate the studies on MIH to determine the consistency of the use of the current definition and scoring systems.

\section{Methods}

A broad search of MEDLINE, EMBASE, ResearchGate, and Google Scholar databases was conducted for the years 2001 to 2015 , using the index terms 'molar-incisor-hypomineralization', 'MIH', 'idiopathic enamel defects in permanent teeth', 'hypomineralized permanent molars' and 'hypomineralized permanent incisors'. The search was limited to English language articles. All abstracts were read and the full text of available relevant articles was then reviewed and data were extracted by two authors independently. The reference list

Correspondence to: Katherine Kula, Diplomate, American Board of Orthodontics, Chair and Jarabak Endowed Professor, Department of Orthodontics and Oral Facial Genetics, Indiana University School of Dentistry, 1121 W. Michigan St., Indianapolis, IN 46202, USA, Tel: 317-278-9915, E-mail: kkula@iu.edu

Key words: molar incisor hypomineralization, enamel defects, scoring system

Received: January 09, 2017; Accepted: January 19, 2017; Published: January 23, 2017 
of key articles was also examined to include any citations that were not located in the search engines.

Of the 175 initial citations, 94 were selected based on abstracts and the remaining articles dealing with generalized enamel defects or inherited dental anomalies were excluded. After reviewing each publication, 52 articles (40 research paper and 12 reviews) relevant to the formulated research question were chosen for inclusion.

Articles were included if the aim was either to describe MIH including a contemporary definition and scoring system or to estimate the prevalence in a certain population excluding articles focusing on generalized or hereditary enamel defects and other age groups.

\section{Results}

Criteria of the 52 articles included in the current review (12 reviews and 40 clinical prevalence studies) are summarized in Tables 1 and 2.

A total of 25 reports used either Weerheijm et al. [1] or Weerheijm

Table 1. Summary of included articles

\begin{tabular}{|c|c|c|c|c|c|c|}
\hline & Study & Year & Study type & Location & Classification & Definition \\
\hline 1 & Weerheijm et al. [1] & 2001 & Short communication & $\begin{array}{l}\text { Amsterdam, The } \\
\text { Netherlands }\end{array}$ & $\begin{array}{l}\text { The aim of this report was to describe the } \\
\text { phenomenon and find a common name for it }\end{array}$ & $\begin{array}{l}\text { Hypomineralization of systemic origin } \\
\text { of } 1-4 \text { permanent first molar, frequently } \\
\text { associated with affected incisors }\end{array}$ \\
\hline 2 & Weerheijm [2] & 2003 & Review & $\begin{array}{l}\text { Amsterdam, The } \\
\text { Netherlands }\end{array}$ & Not provided & Weerheijm et al. [1] \\
\hline 3 & Weerheijm et al. [3] & 2003 & $\begin{array}{l}\text { Review and experts } \\
\text { meeting conclusions }\end{array}$ & $\begin{array}{l}\text { Amsterdam, The } \\
\text { Netherlands }\end{array}$ & $\begin{array}{l}\text { Judgement criteria were set as: } \\
\text { - absence or presence of demarcated opacities; } \\
\text { - PEB; } \\
\text { - atypical restorations; } \\
\text { - extraction of molars due to MIH; } \\
\text { - failure of eruption of a molar or an incisor }\end{array}$ & Weerheijm et al. [1] \\
\hline 4 & William et al. [38] & 2006 & Review & Melbourne, Australia & Weerhejim et al. $[3]$ & Weerheijm et al. [1] \\
\hline 5 & $\begin{array}{l}\text { Mathu-Muju and Wright } \\
\text { [39] }\end{array}$ & 2006 & Chapter & North Carolina, USA & $\begin{array}{l}\text {-Mild MIH: Demarcated opacities in nonstress-bearing } \\
\text { area, isolated, no fracture, no hypersensitivity or caries. } \\
\text {-Moderate MIH: Intact atypical restorations, } \\
\text { demarcated opacities on occlusal/incisal third of teeth } \\
\text { without PEB, PEB or caries limited to } 1 \text { or } 2 \text { surfaces } \\
\text { without cuspal involvement. } \\
\text {-Severe MIH: PEB, history of sensitivity, caries, crown } \\
\text { destruction, defective atypical restoration, esthetic } \\
\text { concerns }\end{array}$ & Weerheijm et al. [1] \\
\hline 6 & Crombie et al. [18] & 2009 & Review & Melbourne, Australia & DDE Index & $\begin{array}{l}\text { Demarcated, qualitative defects of } \\
\text { enamel of systemic origin, affecting } \\
\text { one or more permanent molars } \\
\text { [usually FPMs] with or without } \\
\text { involvement of the incisor teeth }\end{array}$ \\
\hline 7 & $\begin{array}{l}\text { dos Santos and Maia } \\
{[40]}\end{array}$ & 2012 & Chapter & Rio de Janerio, Brazil & $\begin{array}{l}\text { The 10-point scoring system based on the EAPD } \\
\text { criteria (Ghanim et al. [16]) }\end{array}$ & $\begin{array}{l}\text { Morphological enamel defects } \\
\text { involving the occlusal and/or incisal } \\
\text { third of one or more permanent } \\
\text { molars or incisors as result of } \\
\text { hypomineralization of systemic origin }\end{array}$ \\
\hline 8 & $\begin{array}{l}\text { Sadashivamurthy and } \\
\text { Deshmukh [41] }\end{array}$ & 2012 & Review & Karnataka, India & Weerhejim et al. [3] & $\begin{array}{l}\text { Acquired developmental defect } \\
\text { primarily disrupting mineralization of } \\
\text { permanent first molars and incisors }\end{array}$ \\
\hline 9 & Shubha and Hedge [5] & 2013 & Review & Rajasthan, India & Weerheijm et al. [3] & Weerheijm et al. [1] \\
\hline 10 & Seow [42] & 2014 & Review & Brisbane, Australia & Not provided & $\begin{array}{l}\text { A condition where the permanent } \\
\text { molars and incisors show demarcated } \\
\text { areas of hypomineralization or } \\
\text { opacities and often have PEB of the } \\
\text { weak tooth structure and show high } \\
\text { susceptibility to caries }\end{array}$ \\
\hline 11 & Bajwa et al. [43] & 2014 & Review & Andhra Pradesh, India & Weerhejim et al. [3] & Weerheijm et al. [1] \\
\hline 12 & Ghanim et al. [17] & 2015 & Review & Melbourne, Australia & $\begin{array}{l}\text { A detailed diagnostic chart was proposed combining } \\
\text { both clinical presentation of the enamel lesion and } \\
\text { the size of the surface area affected: } \\
0 \text { : no visible enamel defect; } 1 \text { : enamel defect, } \\
\text { non-MIH, 2: White creamy demarcated, yellow or } \\
\text { brown demarcated opacities; } 3 \text { : PEB; } 4 \text { : Atypical } \\
\text { restoration; 5: Atypical caries; } 6: \text { Missing because of } \\
\text { MIH; 7: Cannot be scored. Lesion extension criteria } \\
\text { (after diagnosing i.e. scores } 2 \text { to 6): I: less than one } \\
\text { third of the tooth affected; II: at least one third but } \\
\text { less than two thirds of the tooth affected; III: at least } \\
\text { two thirds of the tooth affected }\end{array}$ & Weerheijm et al. [1] \\
\hline
\end{tabular}

PEB: posteruptive enamel breakdown; PFM: permanent first molar; EAPD: European Academy of Pediatric Dentistry; DDE: developmental defects of enamel index 
Table 2. Summary of included studies.

\begin{tabular}{|c|c|c|c|c|c|c|c|}
\hline & Study & Year & Study type & Location & Sample & Scoring & Definition \\
\hline 1 & $\begin{array}{l}\text { Calderara et al. } \\
{[32]}\end{array}$ & 2005 & Prospective cohort & Lissone, Italy & $\begin{array}{l}\text { Total: } 227 \text { children, } \\
\text { age } 7.3-8.3 \text { years, } \\
\text { MIH: } 13.7 \%\end{array}$ & Weerheijm et al. [1] & Weerheijm et al. [1] \\
\hline 2 & Fteita et al. [22] & 2006 & Prospective cohort & Benghazi, Libya & $\begin{array}{l}188 \text { children, age } \\
7-8.9 \text { years, MIH: } \\
2.9 \%\end{array}$ & $\begin{array}{l}\text {-Mild: color changes of smooth surface } \\
\text {-Moderate: loss of enamel without } \\
\text { dentine involvement } \\
\text {-Severe: lesions affecting both the enamel } \\
\text { and the dentine, atypical restorations } \\
\text { and teeth extracted because of severe } \\
\text { hypomineralization }\end{array}$ & Weerheijm et al. [1] \\
\hline 3 & $\begin{array}{l}\text { Preusser et al. } \\
{[10]}\end{array}$ & 2007 & Prospective cohort & Giessen, Germany & $\begin{array}{l}\text { Total: } 1,002 \\
\text { children, age } 6-12 \\
\text { years, MIH: } 5.9 \%\end{array}$ & $\begin{array}{l}\text { - Degree 1: Isolated white, cream, } \\
\text { yellowish-brown discolorations on the } \\
\text { chewing surface and upper part of the } \\
\text { crown } \\
\text { - Degree 2: hypomineralized yellowish- } \\
\text { brown enamel affecting the humps on the } \\
\text { top of the crown, but with only a slight } \\
\text { loss of substance } \\
\text { - Degree 3: Large-scale mineral } \\
\text { deficiency with distinct yellowish brown } \\
\text { discolorations and defects in crown } \\
\text { morphology resulting from extensive loss } \\
\text { of enamel. }\end{array}$ & $\begin{array}{l}\text { Enamel defects in first } \\
\text { permanent molars and permanent } \\
\text { incisors, ranging from distinct, } \\
\text { isolated, white, and cream- } \\
\text { colored markings to large-scale } \\
\text { ill structuring }\end{array}$ \\
\hline 4 & $\begin{array}{l}\text { Jasulaityte et } \\
\text { al. [44] }\end{array}$ & 2007 & Prospective cohort & Kaunas, Lithuania & $\begin{array}{l}\text { Total: } 1,277 \\
\text { children, age } 7-9 \\
\text { years, MIH: } 9.7 \%\end{array}$ & $\begin{array}{l}\text { MIH cases were categorized into three } \\
\text { groups (opacities, enamel breakdown, } \\
\text { and atypical restorations) and were } \\
\text { recoded into two severity levels: opacities } \\
\text { and disintegration defects. The affected } \\
\text { children were divided into two groups: } \\
\text { children having 1-2 affected teeth and } \\
\text { children having 3-6 affected teeth }\end{array}$ & Weerheijm et al. [1] \\
\hline 5 & $\begin{array}{l}\text { Muratbegovic } \\
\text { et al. }[33]\end{array}$ & 2007 & $\begin{array}{l}\text { Cross-sectional case- } \\
\text { control study }\end{array}$ & $\begin{array}{l}\text { Sarajevo, Bosnia and } \\
\text { Herzegovina }\end{array}$ & $\begin{array}{l}\text { Total: } 560 \text { children, } \\
12 \text { years old, MIH: } \\
12.3 \%\end{array}$ & EAPD [3] & $\begin{array}{l}\text { The systemic hypomineralization } \\
\text { of one, two, three or all four } \\
\text { FPM often followed by changes } \\
\text { of maxillary and somewhat } \\
\text { less commonly of mandibular } \\
\text { permanent incisors }\end{array}$ \\
\hline 6 & $\begin{array}{l}\text { Jasulaityte et } \\
\text { al. [45] }\end{array}$ & 2008 & Prospective cohort & $\begin{array}{l}\text { Amsterdam, The } \\
\text { Netherlands }\end{array}$ & $\begin{array}{l}\text { Total: } 442 \text { children, } \\
\text { age } 6-11 \text { years, } \\
\text { MIH: } 14.3 \%\end{array}$ & EAPD [3] & Weerheijm et al. $[1]$ \\
\hline 7 & $\begin{array}{l}\text { Lygidakis et al. } \\
{[25]}\end{array}$ & 2008 & Prospective cohort & Athens, Greece & $\begin{array}{l}\text { Total: } 3518 \text { children, } \\
\text { age } 5.5-12 \text { years, } \\
\text { MIH: } 10.2 \%\end{array}$ & $\begin{array}{l}\text {-Mild defects: demarcated } \\
\text { opacities } \\
\text {-Moderate/severe defects: PEB and } \\
\text { atypical restorations }\end{array}$ & Weerheijm et al. [1] \\
\hline 8 & Laisi et al. [23] & 2008 & Prospective cohort & Helsinki, Finland & $\begin{array}{l}\text { Total: } 167 \text { children, } \\
\text { age } 7-10 \text { years, } \\
\text { MIH: } 14.4 \%\end{array}$ & $\begin{array}{l}\text {-Mild defects: Demarcated opacity larger } \\
\text { than } 2 \mathrm{~mm} \text { in diameter } \\
\text {-Moderate defects: Broken enamel } \\
\text {-Severe defects: Loss of enamel with } \\
\text { affected dentine, or atypical restoration }\end{array}$ & Weerheijm et al. [1] \\
\hline 9 & $\begin{array}{l}\text { Whatling and } \\
\text { Fearne [46] }\end{array}$ & 2008 & Prospective cohort & London, UK & $\begin{array}{l}\text { Total: } 109 \text { children, } \\
\text { age } 6-13 \text { years, } \\
\text { MIH: } 57 \text { children }\end{array}$ & Not provided & Weerheijm et al. [1] \\
\hline 10 & Cho et al. [12] & 2008 & $\begin{array}{l}\text { Retrospective cross- } \\
\text { sectional }\end{array}$ & Hong Kong & $\begin{array}{l}\text { Total: } 2635 \text { records, } \\
12 \text { years old, MIH: } \\
2.8 \%\end{array}$ & $\begin{array}{l}\text { The severity of hypomineralization was } \\
\text { not recorded }\end{array}$ & Weerheijm et al. [1] \\
\hline 11 & $\begin{array}{l}\text { Kusku et al. } \\
{[47]}\end{array}$ & 2008 & $\begin{array}{l}\text { Retrospective clinical } \\
\text { study }\end{array}$ & Istanbul, Turkey & $\begin{array}{l}\text { Total: } 147 \text { children, } \\
\text { age } 7-9 \text { years, MIH: } \\
14.9 \%\end{array}$ & EAPD [3] & Weerheijm et al. [1] \\
\hline 12 & $\begin{array}{l}\text { Chawla et al. } \\
{[27]}\end{array}$ & 2008 & $\begin{array}{l}\text { Retrospective cross- } \\
\text { sectional }\end{array}$ & Melbourne, Australia & $\begin{array}{l}\text { Records of } 182 \mathrm{MIH} \\
\text { children, age } 6-14 \\
\text { years }\end{array}$ & $\begin{array}{l}\text {-Mild defects: Teeth with white-cream } \\
\text { opacities } \\
\text {-Moderate-severe: Yellow-brown } \\
\text { discoloration or PEB hypomineralization }\end{array}$ & Weerheijm et al. [1] \\
\hline 13 & $\begin{array}{l}\text { Chawla et al. } \\
{[28]}\end{array}$ & 2008 & $\begin{array}{l}\text { Retrospective cross- } \\
\text { sectional }\end{array}$ & Melbourne, Australia & $\begin{array}{l}\text { Records of } 182 \mathrm{MIH} \\
\text { children, age } 6-14 \\
\text { years }\end{array}$ & $\begin{array}{l}\text { Hypomineralization Severity Index. } \\
\text { (Chawla } \text { et al. } 2008 \text { ) } \\
\text { * Presence: FPM unerupted }=0 ; \text { FPM } \\
\text { erupted }=1 . \\
\text { * Extent of hypomineralization: none }=0 ; \\
\text { mild }=1 ; \text { moderate-severe }=2 \text {. } \\
* \text { Sensitivity: none }=0 ; \text { sensitive }=1 . \\
* \text { No. restorative procedures: none }=0 ; \\
\text { one }=1 ; \text { two }=2 ; \text { three or more }=3\end{array}$ & Weerheijm et al. [1] \\
\hline
\end{tabular}




\begin{tabular}{|c|c|c|c|c|c|c|c|}
\hline 14 & Laisi et al. [48] & 2009 & Prospective cohort & Lammi, Finland & $\begin{array}{l}\text { Total: } 141 \text { children, } \\
\text { age } 7.8-12.7 \text { years, } \\
\text { MIH: } 16.3 \%\end{array}$ & $\begin{array}{l}\text { - Mild defects: demarcated opacity } \\
\text {-Severe defects: PEB/atypical restorations } \\
\text { replacing affected enamel }\end{array}$ & Weerheijm et al. [1] \\
\hline 15 & $\begin{array}{l}\text { Kuscu et al. } \\
\text { [49] }\end{array}$ & 2009 & Prospective cohort & Kocaeli, Turkey & $\begin{array}{l}\text { Total: } 153 \text { children, } \\
\text { age } 7-10 \text { years, } \\
\text { MIH: } 9.1-9.2 \%\end{array}$ & EAPD [3] & Weerheijm et al. [1] \\
\hline 16 & Kemoli [26] & 2009 & $\begin{array}{l}\text { Prospective cross- } \\
\text { sectional }\end{array}$ & Nairobi, Kenya & $\begin{array}{l}\text { Total: } 3,591 \\
\text { children, age 6-8 } \\
\text { years, MIH: } 13.73 \%\end{array}$ & $\begin{array}{l}\text { 0: No opacities or discolorations, } \\
\text { 1: Demarcated opacities, PEB, large and } \\
\text { extensive restorations suspected to be a } \\
\text { result of hypomineralization. }\end{array}$ & Weerheijm et al. [1] \\
\hline 17 & $\begin{array}{l}\text { Soviero et al. } \\
{[13]}\end{array}$ & 2009 & Prospective cohort & Rio de Janeiro, Brazil & $\begin{array}{l}\text { Total: } 249 \text { children, } \\
\text { age } 7-13 \text { years, } \\
\text { MIH: } 40.2 \%\end{array}$ & $\begin{array}{l}\text { 0: Absence of demarcated opacity } \\
\text { 1: Presence of a demarcated opacity, PEB, } \\
\text { an atypical restoration, or extraction due } \\
\text { to demarcated opacities }\end{array}$ & $\begin{array}{l}\text { Demarcated opacities which are } \\
\text { qualitative defects involving an } \\
\text { alteration in the translucency of } \\
\text { the enamel, which is otherwise } \\
\text { normal in terms of thickness and } \\
\text { smoothness of its surface often } \\
\text { affecting PFM }\end{array}$ \\
\hline 18 & $\begin{array}{l}\text { da Costa-Silva } \\
\text { et al. }[24]\end{array}$ & 2010 & Prospective cohort & Araraquara-SP, Brasil & $\begin{array}{l}\text { Total: } 918 \text { children, } \\
\text { age } 6-12 \text { years, } \\
\text { MIH: } 19.8 \%\end{array}$ & $\begin{array}{l}\text {-Mild: demarcated opacities larger than } \\
1.0 \mathrm{~mm} \text { with no need of treatment } \\
\text {-Moderate: lesions with rough and } \\
\text { broken enamel } \\
\text {-Severe: loss of dental structure affecting } \\
\text { both enamel and dentin, atypical } \\
\text { restorations replacing affected hard tissue } \\
\text { or teeth extracted because of severe } \\
\text { hypomineralization }\end{array}$ & Weerheijm et al. [1] \\
\hline 19 & $\begin{array}{l}\text { Ghanim et al. } \\
{[16]}\end{array}$ & 2011 & Prospective cohort & Mosul, Iraq & $\begin{array}{l}\text { Total: } 823 \text { children, } \\
\text { age } 7-9 \text { years, MIH: } \\
18.6 \%\end{array}$ & $\begin{array}{l}\text { 0: Enamel defect free; } \\
\text { 1: White/creamy demarcated opacities, } \\
\text { no PEB; 1a: White/creamy demarcated } \\
\text { opacities, with PEB; } \\
\text { 2: Yellow/brown demarcated opacities, } \\
\text { no PEB; 2a: Yellow/brown demarcated } \\
\text { opacities, with PEB; } \\
\text { 3: Atypical restoration; } \\
\text { 4: Missing because of MIH; 5: Partially } \\
\text { erupted with evidence of MIH; } \\
\text { 6: Unerupted/partially erupted with no } \\
\text { evidence of MIH; } \\
\text { 7: Diffuse opacities (not MIH); } \\
\text { 8: Hypoplasia (not MIH); } \\
\text { 9: Combined lesion (diffuse opacities/ } \\
\text { hypoplasia with MIH); } \\
\text { 10: Demarcated opacities in incisors only }\end{array}$ & Weerheijm et al. [1] \\
\hline 20 & $\begin{array}{l}\text { Brogardh-Roth } \\
\text { et al. [11] }\end{array}$ & 2011 & $\begin{array}{l}\text { Cross-sectional case- } \\
\text { control study }\end{array}$ & Malmo, Sweden & $\begin{array}{l}\text { Total: } 82 \text { children, } \\
\text { age } 10-12 \text { years, } \\
\text { MIH: } 38 \%\end{array}$ & $\begin{array}{l}\text { - Mild: Hard, normal surface white, } \\
\text { creamy, yellow or brown defects } \\
\text {-Moderate: Minor loss of substance with } \\
\text { no need of restoration } \\
\text {-Severe: disintegration of enamel and } \\
\text { needed restoration, or atypical restoration }\end{array}$ & $\begin{array}{l}\text { A demarcated opacity with } \\
\text { a diameter exceeding } 2 \mathrm{~mm} \text {, } \\
\text { or PEB/ atypical restoration/ } \\
\text { extraction as a result of MIH in } \\
\text { the permanent first molars }\end{array}$ \\
\hline 21 & $\begin{array}{l}\text { Zawaideh et al. } \\
\text { [35] }\end{array}$ & 2011 & $\begin{array}{l}\text { Prospective cross- } \\
\text { sectional }\end{array}$ & Irbid, Jordan & $\begin{array}{l}\text { Total: } 3241 \text { children, } \\
\text { age } 7-9 \text { years, } \\
\text { MIH: } 17.6 \%\end{array}$ & EAPD $[3]$ & Weerheijm et al. [1] \\
\hline 22 & $\begin{array}{l}\text { Elfrink et al. } \\
{[50]}\end{array}$ & 2012 & Prospective cohort & $\begin{array}{l}\text { Rotterdam, the } \\
\text { Netherlands }\end{array}$ & $\begin{array}{l}\text { Total: } 6161 \text {, age } \\
6-12 \text { years, MIH: } \\
8.7 \%\end{array}$ & EAPD [3] & Weerheijm, et al. [1] \\
\hline 23 & $\begin{array}{l}\text { Ahmadi et al. } \\
{[51]}\end{array}$ & 2012 & Prospective cohort & Zahedan, Iran & $\begin{array}{l}\text { Total: } 433 \text {, age } 7-9 \\
\text { years, MIH: } 12.7 \%\end{array}$ & DDE index & Weerheijm et al. [1] \\
\hline 24 & $\begin{array}{l}\text { Martinez } \\
\text { Gomez et al. } \\
{[52]}\end{array}$ & 2012 & Prospective cohort & Barcelona, Spain & $\begin{array}{l}\text { Total: } 505 \text { children, } \\
\text { age } 6-14 \text { years, } \\
\text { MIH: } 17.85 \%\end{array}$ & Mathu-Muju and Wright [39] & Weerheijm et al. [1] \\
\hline 25 & $\begin{array}{l}\text { Balmer et al. } \\
{[21]}\end{array}$ & 2012 & Prospective cohort & Leeds, UK & $\begin{array}{l}\text { Total: } 3233 \text { children, } \\
12 \text { years old, } \\
\text { MIH: } 15.9 \%\end{array}$ & $\mathrm{mDDE}$ & $\begin{array}{l}\text { A pattern of enamel defects that } \\
\text { consist of asymmetric, } \\
\text { well-demarcated opacities } \\
\text { affecting the FPMs and is } \\
\text { associated with similar defects in } \\
\text { permanent incisors and canines }\end{array}$ \\
\hline 26 & $\begin{array}{l}\text { Parikh et al. } \\
{[53]}\end{array}$ & 2012 & $\begin{array}{l}\text { Prospective cross- } \\
\text { sectional }\end{array}$ & Gujarat, India & $\begin{array}{l}\text { Total: } 1366 \text { children, } \\
\text { age } 8-12 \text { years, } \\
\text { MIH: } 9.2 \%\end{array}$ & $\begin{array}{l}\text {-Mild: demarcated enamel opacities } \\
\text { without enamel breakdown, occasional } \\
\text { sensitivity to external stimuli and only } \\
\text { mild esthetic concerns } \\
\text {-Severe: demarcated enamel opacities } \\
\text { with breakdown, caries, persistent// } \\
\text { spontaneous hypersensitivity affecting } \\
\text { function }\end{array}$ & Weerheijm et al. [1] \\
\hline
\end{tabular}




\begin{tabular}{|c|c|c|c|c|c|c|c|}
\hline 27 & $\begin{array}{l}\text { Souza et al. } \\
\text { [54] }\end{array}$ & 2012 & Prospective cohort & São Paulo, Brazil & $\begin{array}{l}\text { Total: } 903 \text { children, } \\
\text { age } 6-12 \text { years, } \\
\text { MIH: } 24.9 \% \text { and } \\
17.8 \% \text {, }\end{array}$ & EAPD [3] & Weerheijm et al. [1] \\
\hline 28 & $\begin{array}{l}\text { Jeremias et al. } \\
{[55]}\end{array}$ & 2013 & $\begin{array}{l}\text { Cross-sectional } \\
\text { observational }\end{array}$ & São Paulo, Brazil & $\begin{array}{l}\text { Total: } 1157 \text { children, } \\
\text { age } 6-12 \text { years, } \\
\text { MIH: } 12.3 \%\end{array}$ & $\begin{array}{l}\text {-Mild: demarcated enamel opacities of } \\
\text { different colors either white, yellow and } \\
\text { brown } \\
\text {-Severe: PEB, atypical restorations and } \\
\text { extraction due to MIH }\end{array}$ & Weerheijm et al. [1] \\
\hline 29 & $\begin{array}{l}\text { Sönmez et al. } \\
\text { [34] }\end{array}$ & 2013 & Prospective cohort & Ankara, Turkey & $\begin{array}{l}\text { Total: } 4049 \text { children, } \\
\text { age } 7-12 \text { years, } \\
\text { MIH: } 7.7 \%\end{array}$ & EAPD [3] & Weerheijm et al. [1] \\
\hline 30 & $\begin{array}{l}\text { Mittal et al. } \\
{[14]}\end{array}$ & 2014 & $\begin{array}{l}\text { Prospective cross- } \\
\text { sectional }\end{array}$ & Chandigarh, India & $\begin{array}{l}\text { Total: } 1792 \text { children, } \\
\text { age 6-9 years, MIH: } \\
6.31 \%\end{array}$ & $\begin{array}{l}\text { The 10-point scoring system based on the } \\
\text { EAPD criteria (Ghanim et al. }[16] \text { ) }\end{array}$ & Weerheijm et al. [1] \\
\hline 31 & \begin{tabular}{|l|} 
Saldias-Vargas \\
et al. $[56]$
\end{tabular} & 2014 & Prospective cohort & Bauru, Brazil & $\begin{array}{l}\text { Total: } 150 \text {, age } \\
6-12 \text { years, MIH: } \\
4 \%, 22 \%, 42 \% \\
\text { for non-cleft, cleft } \\
\text { palate, and cleft lip } \\
\text { and palate groups } \\
\text { respectively }\end{array}$ & mDDE index & $\begin{array}{l}\text { Enamel defects in PFMs } \\
\text { combined with hypomineralized } \\
\text { incisors }\end{array}$ \\
\hline 32 & $\begin{array}{l}\text { Garcia-Margarit } \\
\text { et al. [57] }\end{array}$ & 2014 & $\begin{array}{l}\text { Prospective cross- } \\
\text { sectional }\end{array}$ & Valencia, Spain & $\begin{array}{l}\text { Total: } 840 \text { children, } \\
8 \text { years old, MIH: } \\
21.8 \%\end{array}$ & $\begin{array}{l}\text { 0-Permanent teeth free of pathological } \\
\text { conditions, } \\
\text { M-MIH } \\
\text { O: Demarcated opacities }>2 \mathrm{~mm} \text { diameter } \\
\text { P: Post-eruption enamel breakdown } \\
\text { C: Extensive caries with opacities around } \\
\text { edge } \\
\text { R: Atypical restorations. } \\
\text { E: Missing permanent first molars as a } \\
\text { result of MIH } \\
\text { N: Tooth not erupted }\end{array}$ & Weerheijm et al. [1] \\
\hline 33 & $\begin{array}{l}\text { Shrestha et al. } \\
\text { [58] }\end{array}$ & 2014 & $\begin{array}{l}\text { Prospective cross- } \\
\text { sectional }\end{array}$ & Kavre, Nepal & $\begin{array}{l}\text { Total: } 749 \text { children, } \\
\text { age } 7-12 \text { years, } \\
\text { MIH: } 13.7 \% \\
\end{array}$ & $\begin{array}{l}\text { 10-point scoring system based on EAPD } \\
\text { criteria (Ghanim } \text { et al. } 2011 \text { ) } \\
\text { Unerupted tooth was considered as } \\
\text { not having MIH; thus code } 6 \text { was also } \\
\text { considered as code } 0 \text {. Also, both the } \\
\text { white/creamy demarcated opacities, } \\
\text { with PEB and yellow/brown demarcated } \\
\text { opacities, with PEB were included in the } \\
\text { group of teeth with PEB }\end{array}$ & Weerheijm et al. [1] \\
\hline 34 & Jankovic [59] & 2014 & $\begin{array}{l}\text { Retrospective cross- } \\
\text { sectional }\end{array}$ & $\begin{array}{l}\text { Foča, Bosnia and } \\
\text { Herzegovina }\end{array}$ & $\begin{array}{l}\text { Total: } 141 \text { children, } \\
8 \text { years old, MIH: } \\
12.8 \% \\
\end{array}$ & $\begin{array}{l}\text {-Mild: Enamel color changes (white, } \\
\text { yellow or brown) } \\
\text {-Moderate: discoloration and minimal } \\
\text { loss of tooth substances without the need } \\
\text { for restoration } \\
\text {-Severe: damaged enamel and dentin loss } \\
\text { that require restoration }\end{array}$ & Weerheijm et al. [1] \\
\hline 35 & $\begin{array}{l}\text { Petrou et al. } \\
{[60]}\end{array}$ & 2014 & Prospective cohort & Greifswald, Germany & $\begin{array}{l}\text { Total: } 2395 \text { children, } \\
\text { age } 8-9 \text { years, MIH: } \\
10.1 \%\end{array}$ & $\begin{array}{l}\text { Mild or severe alteration according to the } \\
\text { degree of tooth structure loss }\end{array}$ & Weerheijm et al. [1] \\
\hline 36 & $\begin{array}{l}\text { Ghanim et al. } \\
\text { [19] }\end{array}$ & 2014 & Prospective cohort & Shiraz, Iran & $\begin{array}{l}\text { Total: } 810 \text { children, } \\
\text { age } 9-11 \text { years, } \\
\text { MIH: } 164(20.2 \%)\end{array}$ & $\begin{array}{l}\text {-Mild: color changes only (i.e. creamy } \\
\text { white or yellow/brown) } \\
\text {-Moderate: loss in enamel substance } \\
\text {-Severe: loss of enamel associated } \\
\text { with affected dentine and/or atypical } \\
\text { restoration }\end{array}$ & $\begin{array}{l}\text { The clinical presence of } \\
\text { developmental defects that } \\
\text { can be seen as opacities, } \\
\text { discoloration or as a mixture } \\
\text { of change in appearance and } \\
\text { loss of enamel substance. The } \\
\text { FPMs and permanent incisors } \\
\text { have been reported as the } \\
\text { most frequently affected teeth, } \\
\text { however, any other tooth, } \\
\text { primary or permanent, may be } \\
\text { affected at the same time }\end{array}$ \\
\hline 37 & $\begin{array}{l}\text { Krishnan et al. } \\
\text { [61] }\end{array}$ & 2015 & Prospective cohort & Tamilnadu, India & $\begin{array}{l}\text { Total: } 4989 \text { children, } \\
\text { age } 9-14 \text { years, } \\
\text { MIH: } 7.3 \%\end{array}$ & $\begin{array}{l}\text { The 10-point scoring system based on the } \\
\text { EAPD criteria (Ghanim } \text { et al. [16]) }\end{array}$ & Weerheijm et al. [1] \\
\hline 38 & $\begin{array}{l}\text { Hussein et al. } \\
\text { [62] }\end{array}$ & 2015 & Prospective cohort & Selangor, Malaysia & $\begin{array}{l}\text { Total: } 154 \text { children, } \\
\text { age } 7-12 \text { years, } \\
\text { MIH: } 16.9 \%\end{array}$ & $\begin{array}{l}\text {-Mild: Enamel color changes (white, } \\
\text { yellow or brown) } \\
\text {-Moderate: discoloration and minimal } \\
\text { loss of tooth substances without the need } \\
\text { for restoration } \\
\text {-Severe: damaged enamel and dentin loss } \\
\text { that require restoration }\end{array}$ & Weerheijm et al. [1] \\
\hline
\end{tabular}




\begin{tabular}{|c|c|c|c|c|c|c|c|}
\hline 39 & $\begin{array}{l}\text { Oyedele } \\
\text { et al. [63] }\end{array}$ & 2015 & $\begin{array}{l}\text { Prospective cross- } \\
\text { sectional }\end{array}$ & Ile-Ife, Nigeria & $\begin{array}{l}\text { Total: } 2,107 \\
\text { children, age } 8-16 \\
\text { years, MIH: } 12.7 \%\end{array}$ & $\begin{array}{l}\text {-Mild: Demarcated opacities present in } \\
\text { the non-stress bearing areas of the molars } \\
\text { with no enamel loss from fracturing } \\
\text {-Moderate: Atypical restoration, } \\
\text { demarcated opacities present on the } \\
\text { occlusal/incisal third of the teeth with no } \\
\text { PEB or with PEB limited to one or two } \\
\text { surfaces without cuspal involvement } \\
\text {-Severe: PEB }\end{array}$ & Weerheijm et al. [1] \\
\hline 40 & $\begin{array}{l}\text { Kevrekidou } \\
\text { et al. }[20]\end{array}$ & 2015 & $\begin{array}{l}\text { Prospective cross- } \\
\text { sectional }\end{array}$ & Thessaloniki, Greece & $\begin{array}{l}\text { Total: } 2335 \text { children, } \\
\text { age } 8-14 \text { years, } \\
\text { MIH: } 21 \%\end{array}$ & $\begin{array}{l}\text {-Mild: Demarcated enamel opacities, } \\
\text { occasional sensitivity to external stimuli, } \\
\text { and only mild esthetic concerns } \\
\text {-Severe: Demarcated enamel opacities } \\
\text { with PEB, hypersensitivity affecting } \\
\text { function, and strong esthetic concerns }\end{array}$ & $\begin{array}{l}\text { The developmental phenomenon } \\
\text { in which one to four PFMs and } \\
\text { possibly incisors appear with } \\
\text { demarcated opacities due to } \\
\text { enamel hypomineralization }\end{array}$ \\
\hline
\end{tabular}

PEB: posteruptive enamel breakdown; PFM: permanent first molar; EAPD: European Academy of Pediatric Dentistry; DDE: developmental defects of enamel index

et al. [3] definition and scoring system. The most commonly used definition in literature, in general, was the original definition created by Weerheijm et al. [1]: "Hypomineralization of systemic origin of 1-4 permanent first molars frequently associated with affected incisors". Weeheijm et al. [3] characterized the hypomineralization as a demarcated opacity, not diffuse. Some authors, however, modified the definition of hypomineralization to include other clinical criteria such as "color changes or ill structuring" or changing the word "systemic origin" into developmental, qualitative, morphological, or asymmetrical $[10,13,18-20]$. Some authors also included other teeth such as the permanent canines, permanent second molars, or primary teeth in the definition $[19,21]$.

While some of the prevalence reports indicated that they used the EAPD criteria or the modified developmental defects of enamel (DDE) index as their classification system, other authors have either added modifications or created their own judging criteria. For example, Fteita et al. [22], Laisi et al. [23], and da Cost-Silva et al. [24] classified their cases into mild, moderate, or severe according to the degree of tooth structure loss. Lygidakis et al. [25] and Kevrekidou et al. [20] suggested that it's better to classify MIH cases into two groups only (mild and severe) combining the previously designed two categories of moderate and severe into one group. Some investigators $[13,26]$ based their data only on a classification of 0 and 1 , where 0 indicates sound teeth or a normal dentition while 1 denotes MIH affected case without reporting grading or severity.

\section{Discussion}

MIH can affect both esthetics and cariogenic susceptibility and is considered to be a global dental problem. It is described by the EAPD as hypomineralization of systemic origin of 1-4 first permanent molars (FPM) frequently associated with affected incisors [3]. Since then, the defining criteria for the condition seem to be the systemic origin and the demarcated opacities in FPM with or without associated incisors. Clinically, MIH presents as opacities of different colors in the affected teeth that will sometimes undergo post-eruptive breakdown due to the porous and weak enamel surface. Accordingly, the condition can represent a serious and challenging clinical management problem. Children with $\mathrm{MIH}$ require higher levels of treatment needs and demonstrate considerable management problems [27-29]. Although most of the published research is epidemiological data, there is still insufficient evidence about the significance of consequences of $\mathrm{MIH}$ and how it may impact the dental healthcare delivery system in general.

Based on these review findings, there seems to be many reasons why the current MIH terminology and criteria are considered questionable and should be addressed:

\section{Teeth involved}

In some reports, demarcated opacities were not limited to the permanent first molar and incisors. Similar lesions have been observed on second primary molars, permanent canines, second permanent molars and premolars [19,21]. Consequently, some authors [30] described the terminology MIH as "misleading" and claimed that this greatly contributes to an under-estimation of the actual defect. They suggested that mineralization defects on all teeth should be recorded in order to reflect the extent of involvement and if sufficient evidence exist that $\mathrm{MIH}$ is not predominantly restricted to molars and incisors, revising the name should be considered.

While most of the studies consider children as having MIH when one or more FPM meet the diagnostic criteria, others [21] indicated that in the absence of FPMs, MIH diagnosis should be based on the anterior teeth. Balmer et al. [31] introduced other terms such as Incisor Hypomineralization (IH) and Molar Hypomineralization (MH). They indicated that the definition of all three conditions $(\mathrm{MIH}, \mathrm{IH}$ and $\mathrm{MH})$ are mutually exclusive and that demarcated opacities named as $\mathrm{IH}$, $\mathrm{MH}$, and deciduous molar incisor hypomineralization (DMH) should all be considered parts of a spectrum that includes MIH given that these defects share characteristics and risk factors which overlap with $\mathrm{MIH}$ and are expected to have the same etiology. The original inclusion of only molars and sometimes incisors was based on the mixed dentition status of the children examined. These teeth form and erupt earlier than the other permanent teeth and could show either early environmental effects or staged genetic effects on tooth formation. We agree with Weerheijm et al. [3] that if examinations of older children could show that more teeth are involved in hypomineralization than the molars and incisors, then a name change would be appropriate.

\section{Lesion size}

The minimum size of lesions included in the diagnosis of MIH is also inconsistent between studies. While some studies included only defects larger than $2 \mathrm{~mm}$ [16,32-34], others have included all hypomineralized lesions regardless of their size [30,35]. The exclusion of smaller defects might have thus contributed to lower prevalence rates in some reports.

\section{Severity indices or scoring systems}

The majority of published literature basically report prevalence data in certain population. Prevalence figures varied considerably. To a great extent, the lack of standardization and heterogeneity of the scoring or characterization systems used in each study probably contributed to the variability. Although a few studies used the current 
EAPD characterizing system (Table 3), some authors have either used some modifications or created their own scoring system which makes it obvious that there is a certain degree of confusion and lack of consensus [3,22-26]. The usefulness of the early EAPD criteria should also be reevaluated due to the fact that it only categorizes the enamel conditions but does not quantify them. Ghanim et al. [17], following the 2014 EAPD Congress in Poland, developed a complex scoring system to quantify the severity of MIH based on the number of involved teeth as well as the type and extent of the enamel defect.

The severity of MIH may differ significantly between individuals and within different teeth of the same individual. The DDE index was implemented to classify the affected teeth according to the type and extent of the defect into 3 categories: demarcated opacities, diffuse opacities, or hypoplasia. Modifications were later proposed to make classification simpler while more specific and meaningful (Table 4).

According to current EAPD criteria, the most extensively used index, diagnosis of MIH includes recording either absence or presence of demarcated opacities, post-eruptive enamel breakdown, atypical restorations, extractions due to MIH and the failure of eruption of a molar or incisor. The use of different scoring systems and severity indices results in confusing and contradictory results. If a case was only scored according to the most severe defect, this may not reflect meaningful results as it may represent involvement of a few or even one tooth whereas the same score may be given to a case with multiple minor defects in another study using summation of scores of all affected teeth. This inconsistency does not allow accurate representation of the

Table 3. Diagnostic criteria for MIH [3].

\begin{tabular}{|l|l|}
\hline Each tooth should be examined for: \\
\hline 1 & $\begin{array}{l}\text { Absence or presence of demarcated opacities } \\
\text { (defect altering the translucency of the enamel) }\end{array}$ \\
\hline 2 & $\begin{array}{l}\text { Posteruptive enamel breakdown (loss of surface } \\
\text { enamel after tooth eruption, usually associated with } \\
\text { a pre-existing opacity) }\end{array}$ \\
\hline 3 & $\begin{array}{l}\text { Atypical restorations (frequently extended to the } \\
\text { buccal or palatal smooth surfaces reflecting the } \\
\text { distribution of hypoplastic enamel) }\end{array}$ \\
\hline 4 & Extracted molars due to MIH \\
\hline 5 & Failure of eruption of a molar or incisor \\
\hline
\end{tabular}

Table 4. Modified DDE index for use in general purpose epidemiological studies [64].

\begin{tabular}{|l|c|}
\hline \multicolumn{1}{|c|}{} & Code \\
\hline Normal & 0 \\
\hline Demarcated opacities: & 1 \\
\hline white/cream & 2 \\
\hline yellow/brown & \\
\hline Diffuse opacities: & 3 \\
\hline Lines & 4 \\
\hline Patchy & 5 \\
\hline Confluent & 6 \\
\hline Confluent/patchy + staining+ loss of enamel & \\
\hline Hypoplasia: & 7 \\
\hline Pits & 8 \\
\hline Missing enamel & 9 \\
\hline Any other defects & \\
\hline Extent of Defect: & 0 \\
\hline Normal & 1 \\
\hline$<1 \backslash 3$ & 2 \\
\hline At least $1 / 3<2 / 3$ & 3 \\
\hline At least $2 / 3$ & \\
\hline
\end{tabular}

patients' overall oral condition, actual restoration needs, and financial requirements.

Children with MIH have higher levels of treatment needs and satisfactory restoration of affected teeth might be very challenging. A study reported that by the age of nine years, children affected with enamel hypomineralization required dental treatment about ten times more frequently compared to unaffected children and that each hypomineralized tooth was treated on average twice [36]. Actual management needs were underestimated when considering only decayed or restored teeth as most of these children would also require continued pediatric restorative, orthodontic and preventive care [37,38]. This highlights the importance of directing future research to high-quality studies that help to improve our understanding of the exact causes of $\mathrm{MIH}$, allow development of standardized diagnostic criteria and universal severity index, and update the dental profession on the overall impact on the oral health care delivery system.

\section{Conclusions}

Although MIH seems to be a significant dental public health concern, there is insufficient evidence regarding how accurate the currently used definition and judgement criteria reflect the clinical challenges, treatment needs, and cost associated with the condition. Studies continue to be published using alternative definitions and scoring systems and there is lack of agreement on important criteria. Such inconsistency makes it difficult to compare studies and develop evidence based guidelines. A globally accepted definition and scoring system is necessary.

\section{References}

1. Weerheijm KL, Jalevik B, Alaluusua S (2001) Molar-incisor hypomineralisation. Caries Res 35: 390-391. [Crossref]

2. Weerheijm KL (2003) Molar incisor hypomineralisation (MIH). Eur J Paediatr Dent 4: 114-120. [Crossref]

3. Weerheijm K, Duggal M, Mejare I, Papagiannoulis L, Koch G, et al. (2003) Judgement criteria for molar incisor hypomineralisation (MIH) in epidemiologic studies: a summary of the European meeting on MIH held in Athens, 2003. Eur J Paediatr Dent 4: 110-113. [Crossref]

4. Alaluusua S (2010) Aetiology of molar-incisor hypomineralisation: a systematic review. Eur Arch Paediatr Dent 11: 53-58. [Crossref]

5. Shubha AB, Hegde S (2013) Molar incisor hypomineralization: Review of its prevalence, etiology, clinical appearance and management. Int J Oral Maxillofac Path 4: $26-33$

6. Brook AH (2009) Multilevel complex interactions between genetic, epigenetic and environmental factors in the aetiology of anomalies of dental development. Arch Oral Biol 54: S3-17. [Crossref]

7. Whatling R, Fearne JM (2008) Molar incisor hypomineralization: a study of aetiological factors in a group of UK children. Int J Paediatr Dent 18: 155-162. [Crossref]

8. Jeremias F, Koruyucu M, Küchler EC, Bayram M, Tuna EB, et al. (2013) Genes expressed in dental enamel development are associated with molar-incisor hypomineralization. Arch Oral Biol 58: 1434-1442. [Crossref]

9. Kühnisch J, Thiering E, Heitmüller D, Tiesler CM, Grallert H, et al. (2014) Genomewide association study (GWAS) for molar-incisor hypomineralization (MIH). Clin Oral Invest 18: 677-682. [Crossref]

10. Preusser SE, Ferring V, Wleklinski C, Wetzel WE (2007) Prevalence and severity of molar incisor hypomineralization in a region of Germany -- a brief communication. $J$ Public Health Dent 67: 148-150. [Crossref]

11. Brogardh-Roth S, Matsson L, Klingberg G (2011) Molar-incisor hypomineralization and oral hygiene in 10- to-12-yr-old Swedish children born preterm. Eur J Oral Sci 119: 33-39. [Crossref]

12. Cho SY, Ki Y, Chu V (2008) Molar incisor hypomineralization in Hong Kong Chinese children. Int J Paediatr Dent 18: 348-352. [Crossref] 
13. Soviero V, Haubek D, Trindade C, Da Matta T, Poulsen S (2009) Prevalence and distribution of demarcated opacities and their sequelae in permanent 1st molars and incisors in 7 to 13-year-old Brazilian children. Acta Odontol Scand 67: 170-175. [Crossref]

14. Mittal NP, Goyal A, Gauba K, Kapur A (2014) Molar incisor hypomineralisation: prevalence and clinical presentation in school children of the northern region of India. Eur Arch Paediatr Dent 15: 11-18. [Crossref]

15. Elfrink MEC, Ghanim A, Manton DJ, Weerheijm KL (2015) Standardized studies on molar incisor hypomineralisation (MIH) and hypomineralised second primary molars (HSPM): a need. Eur Arch Paediatr Dent 16: 247-255. [Crossref]

16. Ghanim A, Morgan M, Marino R, Bailey D, Manton D (2011) Molar-inciso hypomineralisation: prevalence and defect characteristics in Iraqi children. Int $J$ Paediat Dent 21: 413-421. [Crossref]

17. Ghanim A, Elfrink M, Weerheijm K, Mariño R, Manton D (2015) A practical method for use in epidemiological studies on enamel hypomineralisation. Eur Arch Paediatr Dent 16: 235-246. [Crossref]

18. Crombie F, Manton D, Kilpatrick N (2009) Aetiology of molar-incisor hypomineralization: a critical review. Int J Paediatr Dent 19: 73-83. [Crossref]

19. Ghanim A, Bagheri R, Golkari A, Manton D (2014) Molar-incisor hypomineralisation: a prevalence study amongst primary schoolchildren of Shiraz, Iran. Eur Arch Paediatr Dent 15: 75-82. [Crossref]

20. Kevrekidou A, Kosma I, Arapostathis K, Kotsanos N (2015) Molar incisor hypomineralization of eight-and 14-year-old children: Prevalence, severity, and defect characteristics. Pediatr Dent 37: 455-461. [Crossref]

21. Balmer R, Toumba J, Godson J, Duggal M (2012) The prevalence of molar incisor hypomineralisation in Northern England and its relationship to socioeconomic status and water fluoridation. Int J Paediatr Dent 22: 250-257. [Crossref]

22. Fteita D, Ali A, Alaluusua S (2006) Molar-incisor hypomineralization (MIH) in a group of school-aged children in Benghazi, Libya. Eur Arch Paediatr Dent 7: 92-95. [Crossref]

23. Laisi S, Kiviranta H, Lukinmaa PL, Vartiainen T, Alaluusua S (2008) Molar-incisorhypomineralisation and dioxins: new findings. Eur Arch Paediatr Dent 9: 224-227. [Crossref]

24. da Costa-Silva CM, Jeremias F, de Souza JF, Cordeiro Rde C, Santos-Pinto L, et al. (2010) Molar incisor hypomineralization: prevalence, severity and clinical consequences in Brazilian children. Int J Paediatr Dent 20: 426-434. [Crossref]

25. Lygidakis NA, Dimou G, Briseniou E (2008) Molar-incisor-hypomineralisation (MIH): Retrospective clinical study in Greek children. I. Prevalence and defect characteristics. Eur Arch Paediatr Dent 9: 200-206. [Crossref]

26. Kemoli AM (2008) Prevalence of molar incisor hypomineralisation in six to eight yearolds in two rural divisions in Kenya. East Afr Med J 85: 514-519. [Crossref]

27. Chawla N, Messer LB, Silva M (2008) Clinical studies on molar-incisorhypomineralisation part 1: distribution and putative associations. Eur Arch Paediatr Dent 9: 180-190. [Crossref]

28. Chawla N, Messer LB, Silva M (2008) Clinical studies on molar-incisorhypomineralisation part 2: development of a severity index. Eur Arch Paediatr Dent 9: 191-199. [Crossref]

29. Crombie FA, Manton DJ, Weerheijm KL, Kilpatrick NM (2008) Molar incisor hypomineralization: a survey of members of the Australian and New Zealand Society of Paediatric Dentistry. Aust Dent J 53: 160-166. [Crossref]

30. Lygidakis NA, Wong F, Jälevik B, Vierrou AM, Alaluusua S, et al. (2010) Best clinical practice guidance for clinicians dealing with children presenting with molar-incisorhypomineralisation (MIH): An EAPD policy document. Eur Arch Paediatr Dent 11: 75-81. [Crossref]

31. Balmer R, Toumba KJ, Munyombwe T, Godson J, Duggal MS (2015) The prevalence of incisor hypomineralisation and its relationship with the prevalence of molar incisor hypomineralisation. Eur Arch Paediatr Dent 16: 265-269. [Crossref]

32. Calderara PC, Gerthoux PM, Mocarelli P, Lukinmaa PL, Tramacere PL, et al. (2005) The prevalence of Molar Incisor Hypomineralisation (MIH) in a group of Italian school children. Eur J Paediatr Dent 6: 79-83. [Crossref]

33. Muratbegovic A, Markovic N, Selimovic MG (2007) Molar incisor hypomineralisation in Bosnia and Herzegovina: prevalence, aetiology and clinical consequences in medium caries activity population. Eur Arch Paediatr Dent 8: 189-194. [Crossref]
34. Sonmez H, Yildirim G, Bezgin T (2013) Putative factors associated with molar incisor hypomineralisation: an epidemiological study. Eur Arch Paediatr Dent 14: 375-380. [Crossref]

35. Zawaideh FI, Al-Jundi SH, Al-Jaljoli MH (2011) Molar incisor hypomineralisation: prevalence in Jordanian children and clinical characteristics. Eur Arch Paediatr Dent 12: 31-36. [Crossref]

36. Jalevik B, Klingberg GA (2002) Dental treatment, dental fear and behavior management problems in children with severe enamel hypomineralization of their permanent first molars. Int J Paediatr Dent 12: 24-32. [Crossref]

37. Leppäniemi A, Lukinmaa PL, Alaluusua S (2001) Nonfluoride hypomineralizations in the permanent first molars and their impact on the treatment need. Caries Res 35: 3640. [Crossref]

38. William V, Messer LB, Burrow MF (2006) Molar incisor hypomineralization: review and recommendations for clinical management. Pediatr Dent 28: 224-232. [Crossref]

39. Mathu-Muju K, Wright JT (2006) Diagnosis and treatment of molar incisor hypomineralization. Compend Contin Educ Dent 27: 604-610. [Crossref]

40. dos Santos MPA, Maia LC. Molar incisor hypomineralization: Morphological, aetiological, epidemiological and clinical considerations. INTECH Open Acces Publisher, 2012.

41. Sadashivamurthy P, Deshmukh S (2012) Missing links of molar incisor hypomineralization: a review. J Int Oral Health 4: 1-11.

42. Seow WK (2014) Developmental defects of enamel and dentine: challenges for basic science research and clinical management. Aust Dent J 59: 143-154. [Crossref]

43. Bajwa NK, Jingarwar MM, Pathak A (2014) Molar incisor hypomineralization. Int J Experiment Dent Sci 3: 37-40.

44. Jasulaityte L, Veerkamp JS, Weerheijm KL (2007) Molar incisor hypomineralization: review and prevalence data from a study of primary school children in Kaunas (Lithuania). Eur Arch Paediatr Dent 8: 87-94. [Crossref]

45. Jasulaityte L, Weerheijm KL, Veerkamp JS (2008) Prevalence of molar-incisorhypomineralisation among children participating in the Dutch National Epidemiological Survey. Eur Arch Paediatr Dent 9: 218-223. [Crossref]

46. Whatling R, Fearne JM (2008) Molar incisor hypomineralization: a study of aetiological factors in a group of UK children. Int J Paediatr Dent 18: 155-162. [Crossref]

47. Kusku OO, Caglar E, Sandalli N (2008) The prevalence and aetiology of molar-incisor hypomineralisation in a group of children in Istanbul. Eur J Paediatr Dent 9: 139-144. [Crossref]

48. Laisi S, Ess A, Sahlberg C, Arvio P, Lukinmaa PL, et al. (2009) Amoxicillin may cause molar incisor hypomineralization. J Dent Res 88: 132-136. [Crossref]

49. Kuscu OO, Caglar E, Aslan S, Durmusoglu E, Karademir A, et al. (2009) The prevalence of molar incisor hypomineralization (MIH) in a group of children in a highly polluted urban region and a windfarm-green energy island. Int J Paediatr Dent 19: 176-185. [Crossref]

50. Elfrink ME, ten Cate JM, Jaddoe VW, Hofman A, Moll HA, et al. (2012) Deciduous molar hypomineralization and molar incisor hypomineralization. J Dent Res 91: 551555. [Crossref]

51. Ahmadi R, Ramazani N, Nourinasab R (2012) Molar incisor hypomineralization: a study of prevalence and etiology in a group of Iranian children. Iran J Pediatr 22: 245-251. [Crossref]

52. Martinez Gomez, TP, Guinot F, Bellet LJ, Giner L (2012) Prevalence of molar-inciso hypomineralisation observed using transillumination in a group of children from Barcelona (Spain). Int J Paediatr Dent 22: 100-109. [Crossref]

53. Parikh DR, Ganesh M, Bhaskar V (2012) Prevalence and characteristics of molar incisor hypomineralisation (MIH) in the child population residing in Gandhinagar, Gujarat, India. Eur Arch Paediatr Dent 13: 21-26. [Crossref]

54. Souza JF, Costa-Silva CM, Jeremias F, Santos-Pinto L, Zuanon AC, et al. (2012) Molar incisor hypomineralisation: possible aetiological factors in children from urban and rural areas. Eur Arch Paediatr Dent 13: 164-170. [Crossref]

55. Jeremias F, de Souza JF, Silva CM, Cordeiro Rde C, Zuanon AC, et al. (2013) Dental caries experience and Molar-Incisor Hypomineralization. Acta Odontol Scand 71: 870876. [Crossref]

56. Saldias-Vargas VP, Tovani-Palone MR, Moura-Martins AP, da Silva Dalben G, RibeiroGomide M (2014) Enamel defects in permanent first molars and incisors in individuals 
with cleft lip and/or palate. Revista de la Facultad de Medicina 62: 515-519.

57. Garcia-Margarit M, Catalá-Pizarro M, Montiel-Company JM, Almerich-Silla JM (2014) Epidemiologic study of molar-incisor hypomineralization in 8-year-old Spanish children. Int J Paediatr Dent 24: 14-22. [Crossref]

58. Shrestha R, Upadhaya S2, Bajracharya M2 (2014) Prevalence of molar incisor hypomineralisation among school children in Kavre. Kathmandu Univ Med J (KUMJ) 12: 38-42. [Crossref]

59. Janković S, Ivanović M, Davidović B, Lecić J. (2014) Distribution and characteristics of molar-incisor hypomineralization. Vojnosanit Pregl 71: 730-734. [Crossref]

60. Petrou MA, Giraki M, Bissar AR, Basner R, Wempe C, et al. (2014) Prevalence of molar incisor hypomineralisation among school children in four German cities. Int $J$ Paediatr Dent. 24: 434-440. [Crossref]
61. Krishnan R, Ramesh M, Chalakkal P (2015) Prevalence and characteristics of MIH in school children residing in an endemic fluorosis area of India: an epidemiological study. Eur Arch Paediatr Dent 16: 455-460. [Crossref]

62. Hussein AS, Faisal M, Haron M, Ghanim AM, Abu-Hassan MI (2015) Distribution of molar incisor hypomineralization in Malaysian children attending University Dental Clinic. J Clin Pediatr Dent 39: 219-223. [Crossref]

63. Oyedele TA, Folayan MO, Adekoya-Sofowora CA, Oziegbe EO (2015) Co-morbidities associated with molar-incisor hypomineralisation in 8 to 16 year old pupils in Ile-Ife, Nigeria. BMC Oral Health 15: 37. [Crossref]

64. Clarkson J, O’Mullane D (1989) A modified DDE Index for use in epidemiological studies of enamel defects. $J$ Dent Res 68: 445-450. [Crossref]

Copyright: $\odot 2017$ Allam E. This is an open-access article distributed under the terms of the Creative Commons Attribution License, which permits unrestricted use, distribution, and reproduction in any medium, provided the original author and source are credited. 